\title{
Conservation of Genetically-Embedded Virus Assembly Instructions: A Novel Route to Antiviral Therapy ${ }^{+}$
}

\author{
Reidun Twarock 1,2,3,*, Peter G. Stockley ${ }^{4}$, Richard J. Bingham 1,2,3, Eric C. Dykeman 1,3 \\ and Pierre-Philippe Dechant ${ }^{5}$ \\ 1 Departments of Mathematics and Biology and York Cross-disciplinary Centre for Systems Analysis, \\ University of York, York YO10 5DD, UK; r.j.bingham@york.ac.uk (R.J.B.); \\ eric.dykeman@york.ac.uk (E.C.D.) \\ 2 Department of Biology, University of York, York YO10 5DD, UK \\ 3 York Cross-disciplinary Centre for Systems Analysis, University of York, York YO10 5DD, UK \\ 4 Astbury Centre for Structural Molecular Biology, Faculty of Biological Sciences, University of Leeds, \\ Leeds LS2 9JT, UK; p.g.stockley@leeds.ac.uk \\ 5 School of Psychological \& Social Sciences, York St John University, York YO31 7EX, UK; \\ p.dechant@yorksj.ac.uk \\ * Correspondence: reidun.twarock@york.ac.uk \\ † Presented at Viruses 2020-Novel Concepts in Virology, Barcelona, Spain, 5-7 February 2020. \\ Published: 18 June 2020
}

\begin{abstract}
Many single-stranded RNA viruses, including major viral pathogens, present RNAencoded virus assembly instructions (VAIs) within their genetic message that can be isolated from the genetic code and repurposed for the design of virus-like particles. These VAIs rely on multiple dispersed RNA secondary structure elements with a consensus recognition motif for the capsid (core) protein, called packaging signals (PSs), which collectively promote capsid assembly. In this talk, I will provide evidence for the evolutionary conservation of the PS-encoded assembly instructions among different viruses in a viral family and discuss the implications of this discovery for viral evolution. I will then demonstrate how the VAIs can be exploited for therapy. In particular, defective interfering particles occur spontaneously in viral evolution as mutant strains lacking essential parts of the viral genome. Their ability to replicate in the presence of wild-type virus at the expense of virally produced resources can be mimicked by therapeutic interfering particles (TIPs). I will introduce a novel approach to the design of such TIPs based on synthetic nucleic acid sequences containing the VAIs but otherwise lacking genetic information. Using multiscale models of a viral infection, I will demonstrate the potential of these particles in both the prophylaxis and treatment of RNA viral infections.
\end{abstract}

Keywords: viral assembly; packaging signals; viral evolution; antiviral therapy; multiscale models; RNA SELEX; Cryo-electron microscopy; bioinformatics

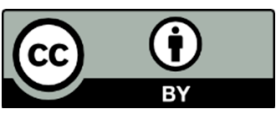

(C) 2020 by the authors. Licensee MDPI, Basel, Switzerland. This article is an open access article distributed under the terms and conditions of the Creative Commons Attribution (CC BY) license (http://creativecommons.org/licenses/by/4.0/). 